\title{
Distribution of Growth Cones and Synapses in Developing Laminar and Interlaminar Regions of the Dorsal Lateral Geniculate Nucleus
}

\author{
Judy K. Brunso-Bechtold and Sherry L. Vinsant \\ Department of Anatomy, Bowman Gray School of Medicine, Winston-Salem, North Carolina 27103
}

In the present study, we quantified the distribution of growth cones and synapses in $\mathbf{2}$ developing layers, as well as in the intervening interlaminar space of the dorsal lateral geniculate nucleus (dLGN) in tree shrews. Our goal was to gain insight into mechanisms involved in the segregation of dLGN cells into layers during development. We sacrificed tree shrews before (P0), during (P4 and P7), and after (P15) laminar segregation as well as at maturity $(P 90)$. The dLGN from each animal was sectioned horizontally, and all tissue for analysis was blocked from the middle third of the nucleus along the dorsoventral axis. Each micrograph was coded and blindly scored for the number of growth cones and synapses in layers 4 and 5 and the intervening interlaminar space. We also measured each growth cone and classified synapse type. Statistical analyses of these data reveal that neither growth cones nor synapses are significantly more common in the interlaminar space early in the period of laminar segregation (P4). By nearly a week after the interlaminar space can first be distinguished (P7), there are more growth cones in the interlaminar space than in the layers, but this difference is no longer present at (P15). These results suggest that, although neuropil development at the laminar borders may not play a role in the onset of laminar segregation, it may contribute to the widening of the interlaminar spaces once this process has begun. In addition, growth cones continuously decrease in number and become less bulbous and more linear in shape with development. Synapses, on the other hand, continuously increase in number with age and pass through a transient period characterized by heavy spinous terminations.

In all species studied, cell layers that develop in the dorsal lateral geniculate nucleus (dLGN) segregate from an unlaminated mass of undifferentiated cells (Rakic, 1977; Linden et al., 1981; Brunso-Bechtold and Casagrande, 1982; Cusik and Kaas, 1982; Shatz, 1983). This lamination takes place after the segregation of the retinal input into eye-specific bands is well underway. If that input is removed bilaterally at birth, the laminar segregation of

\footnotetext{
Received Mar. 13, 1987; revised Nov. 9, 1987; accepted Nov. 11, 1987.

We gratefully acknowledge the excellent technical assistance of Constance Linville, the editorial comments of Dr. Ron Oppenheim and Keith Sutton, the statistical assistance of Dr. Doug Case, Department of Biometry, and manuscript preparation by Sarah Graham. Supported by EYO 5028 tu J.B-B.

Correspondence should be addressed to Judy K. Brunso-Bechtold, Ph.D., Department of Anatomy, Bowman Gray School of Medicine, 300 South Hawthorne Road, Winston-Salem, NC 27103

Copyright C 1988 Society for Neuroscience $0270-6474 / 88 / 082677-16 \$ 02.00 / 0$
}

dLGN cells does not take place, although laminar borders can still be distinguished on the basis of cytological criteria (BrunsoBechtold and Casagrande, 1981; Guillery et al., 1985). Furthermore, in the absence of retinal input, the extraretinal projections to the dLGN in tree shrews no longer terminate in the laminated pattern seen in normal animals (Brunso-Bechtold et al., 1983; Sutton and Brunso-Bechtold, 1988). Of particular interest is the corticogeniculate projection. This projection grows into the dLGN during the first postnatal weeks and normally terminates densely in the interlaminar spaces as the layers are beginning to segregate (Brunso-Bechtold and Casagrande, 1983). However, following removal of retinal input at birth, the corticogeniculate projection no longer terminates at the laminar borders and instead terminates in a homogeneous but topographic band across the mediolateral extent of the nucleus.

What clues do these experimental results provide to the mechanisms normally involved in the redistribution of dLGN cells into layers? One possibility lies in the effect of the partial deafferentation of dLGN cells following removal of retinal input. Deafferentation is known to result in incomplete dendritic development (e.g., see also Cowan, 1970; Peusner and Morest, 1977; Deitch and Rubel, 1984), and dendritic elaborations have becn reported to be a major component of the interlaminar spaces in cat dLGN (Guillery, 1966). It is possible that in the absence of retinal input dLGN dendrites, which normally extend into the interlaminar spaces and receive the corticogeniculate input, fail to develop fully. In the absence of their usual target and in the presence of vacated retinogeniculate synaptic sites, the corticogeniculate fibers would then terminate within the layers rather than at the laminar borders. Thus, following removal of the retinal fibers at birth, the cell layers may fail to segregate because the normal complement of developing dLGN dendrites and ingrowing corticogeniculate fibers are no longer physically pushing the layers apart at the laminar borders.

In normal development, then, the dLGN layers would segregate because of a directed dendritic growth at the laminar borders in combination with an initial ingrowth and subsequent termination of extraretinal projections at the laminar borders. According to this hypothesis, there normally would be more axonal and/or dendritic growth cones in the interlaminar spaces as those spaces are beginning to appear than in the layers. To test this hypothesis, we have quantified the number of growth cones in $2 \mathrm{dLGN}$ layers and in the intervening interlaminar space during the period of laminar segregation. We have also determined the synapse distribution in those areas to reveal any role that temporal or regional variations in synaptogenesis might play in laminar segregation. A preliminary report of these results has appeared in abstract form (Vinsant and Brunso-Bechtold, 1986). 


\section{Materials and Methods}

Tree shrew dLGN anatomy. The dLGN of tree shrews contains 6 layers, numbered from medial to lateral, which are most easily distinguished in horizontal section. Layers $2-4$ and 6 receive input from the contralateral eye, and layers 1 and 5 receive input from the ipsilateral eye. These cell layers form postnatally. By postnatal day $2^{\prime}$ (P2), layers 1 and 2 and layers 4 and 5 have begun to segregate from each other; by P6, the segregation of other layers is underway as well; by P14, all interlaminar spaces are distinguishable but continue to widen as the nucleus matures (see Brunso-Bechtold and Casagrande, 1982).

It obviously would be a prohibitively large task to quantify the growth cone and synapse distribution in all 6 layers of the dLGN and the intervening interlaminar spaces. We therefore selected layers 4 and 5 and the intervening interlaminar space for our analysis. We chose those particular layers for our sample for 2 reasons. First, they allow comparison between a contralaterally innervated layer (layer 4) and an ipsilaterally innervated one (layer 5 ). Second, the interlaminar space between layers 4 and 5, which can first be distinguished by $P 2$, widens rapidly and is large enough for reliable sampling by $\mathrm{P} 4$.

Tissue preparation. In order to study the distribution of growth cones and synapses during laminar segregation, we sacrificed tree shrews before (P0), during (P4 and P7), and just after (P15) laminar segregation, as well as at maturity (P90). Two animals were sacrificed at each age. Each animal was overdosed with sodium pentobarbital $(80 \mathrm{mg} / \mathrm{kg})$ and transcardially perfused for $30-45$ min with a fixative consisting of $2 \%$ glutaraldehyde and $2 \%$ paraformaldehyde in $0.15 \mathrm{~m}$ sodium cacodylate buffer. A peristaltic pump (Ismatec) was used to achieve a constant perfusion rate $(20 \mathrm{ml} / \mathrm{min})$. Following perfusion the animal was decapitated just caudal to the obex, the skull was carefully opened, and the brain was stored overnight in fixative at $4^{\circ} \mathrm{C}$.

The brain then was removed from the cranium and trimmed to include only the diencephalon and rostral mesencephalon. That block of tissue was then sectioned horizontally on a vibratome (Lancer) at 200 $\mu \mathrm{m}$ and collected in $0.15 \mathrm{M}$ sodium cacodylate buffer. The sections containing the dLGN were identified using a Nikon Zoom dissecting microscope mounted on a dark-field/light-field base. The middle third of the dLGN sections (along the dorsoventral axis) were selected, and the dLGN was cut out and placed in labeled vials containing $0.15 \mathrm{M}$ sodium cacodylate buffer. Using the dark-field/light-field illumination, it was possible to cut out blocks whose borders preciscly corresponded to those of the dLGN in all animals and to those of the binocular segment of the dLGN in animals with laminated dLGNs. The blocks were washed in buffer, postfixed for a half-hour in $2 \%$ osmium, washed thoroughly in buffer, and embedded in Araldite. Prompt processing of tissue appears to be important for membrane preservation, especially at the youngest ages. Thin sections ( $700 \AA$ ) were cut on an LKB 4800 ultramicrotome and collected onto Formvar-coated copper slot grids. At the beginning and end of each series of thin sections, a $1 \mu \mathrm{m}$ semithin section was cut with a glass knife and stained with toluidine blue.

Data collection and analysis. Thin sections were viewed with an Hitachi $600 \mathrm{CX}$ electron microscope equipped with micrometers on the $\mathrm{X}$ and $\mathrm{Y}$ stage controls. Each thin section to be used for quantification was reconstructed by recording the $\mathrm{X}$ and $\mathrm{Y}$ coordinates of its perimeter and major landmarks and plotting these onto graph paper. One of the adjacent semithin sections was then projected onto the thin section outline using a zoom camera lucida attached to a Nikon Optiphot light microscope, and laminar borders were drawn onto the outline on the basis of the cytoarchitectural features in the semithin section. At P4, P7, P15, and P90, 12 laminar (6 in layer 4 and 6 in layer 5), and 13 interlaminar sites for electron micrographs were then chosen on the basis of the cytoarchitectural features and indicated on the outline drawing. At P0 (before laminar segregation has begun), 25 sites were chosen randomly from the lateral half of the nucleus, but nonadjacent to the optic tract; this approximates the region of future layers 4 and 5 and the intervening space. The appropriate $X$ and $Y$ micrometer readings were determined for each electron micrograph site indicated on the outline drawing.

\footnotetext{
We count the first day of life as postnatal day $0(\mathrm{P} 0)$, the second day as $\mathrm{P} 1$, and so on.

When using microscope coordinates to graph outline, the X stage micrometer could be read directly and recorded as the $X$ coordinate. The $Y$ stage control on the Hitachi $600 \mathrm{CX}$, however, is not linear with respect to the $\mathrm{X}$ stage control; therefore, each $\mathrm{Y}$ stage micrometer reading was multiplied by 1.25 to obtain the proper $Y$ coordinate. When determining coordinates from graph paper to be used
}

This method accurately relates our EM sample to the light microscopic cytoarchitectural criteria commonly used to identify layers and interlaminar spaces, which are difficult, if not impossible, to distinguish with EM. It also eliminates experimenter bias (e.g., selection on the basis of the presence of cell bodies or a predominance of a certain synaptic type), since the coordinates for each electron micrograph are predetermined.

Each electron micrograph was then coded and scored blindly. For each micrograph the number of growth cones was recorded, and these were broken down into pre- and postsynaptic categories when possible. In addition, synapses were categorized by type of terminal and postsynaptic element and counted. When all counts were completed, the numbers in each category were summed for all interlaminar micrographs, for all layer 4 micrographs and for all layer 5 micrographs per animal. Growth cone areas and perimeters were measured in all micrographs using a Bioquant (R\&M Biometrics) morphometrics system. Shape factors were derived for growth cones using the formula $4 \pi(A) /$ $p^{2}$. The areas of all cell bodies and blood vessels included in the micrographs were also measured, summed, and subtracted from the total area to determine the actual area of neuropil sampled. Growth cone number was subsequently normalized to $10,000 \mu \mathrm{m}^{2}$ of neuropil.

Numbers for statistical analyses were derived from counts in $25 \mathrm{mi}$ crographs per animal and 2 animals per time point. For the effect of age alone, $p$ values were computed using a 1 -way analysis of variance. When assessing age by location effects, the repeated measures analysis of variance model was used. If this test indicated a significant interaction, 2-sided paired $t$ tests were performed at individual time points to reveal effect of location.

\section{Results}

\section{Laminar data}

Our primary intent in the present study was to determine the quantitative differences in the neuropil between the interlaminar spaces and layers. To eliminate any bias introduced by a developmental difference between ipsilaterally and contralaterally innervated dLGN layers, we included both an ipsilaterally innervated layer (layer 5) and a contralaterally innervated one (layer 4) in our sample. Analysis of variance indicates that there is no significant difference in either growth cone number or synapse number between the 2 layers. Therefore, for the results presented here, the data from layers 4 and 5 were combined and reanalyzed for comparison of interlaminar versus laminar data in each category at each age.

\section{Criteria for quantitative categories}

For consistency in quantification of growth cones and synapses, we selected a strict set of morphological features to serve as criteria for categorization. Such criteria are based on features that appear to be distinguishing characteristics of the structures in question. For clarity, the results as well as the descriptions of the morphological features used as criteria will be presented separately for growth cones and synapses.

\section{Growth cones}

Morphology. The asterisks in Figure 1 indicate examples of typical growth cones at $\mathrm{P} 0$ (Fig. $1 A$ ), $\mathrm{P} 4$ (Fig. 1B), P7 (Fig. 1C), and P15 (Fig. $1 D$ ). Although growth cones vary in appearance, several characteristics were consistently present and were used as criteria in identifying profiles as growth cones. Profiles counted as growth cones were at least $0.5 \mu \mathrm{m}^{2}$ in area, were filled with

on microscope, the micrometer reading for the $\mathrm{X}$ stage control could be read directly as the $X$ coordinate on the graph paper; the micrometer reading for the $\mathrm{Y}$ stage control was obtained by dividing the $\mathrm{Y}$ coordinate on the graph paper by 1.25 . 

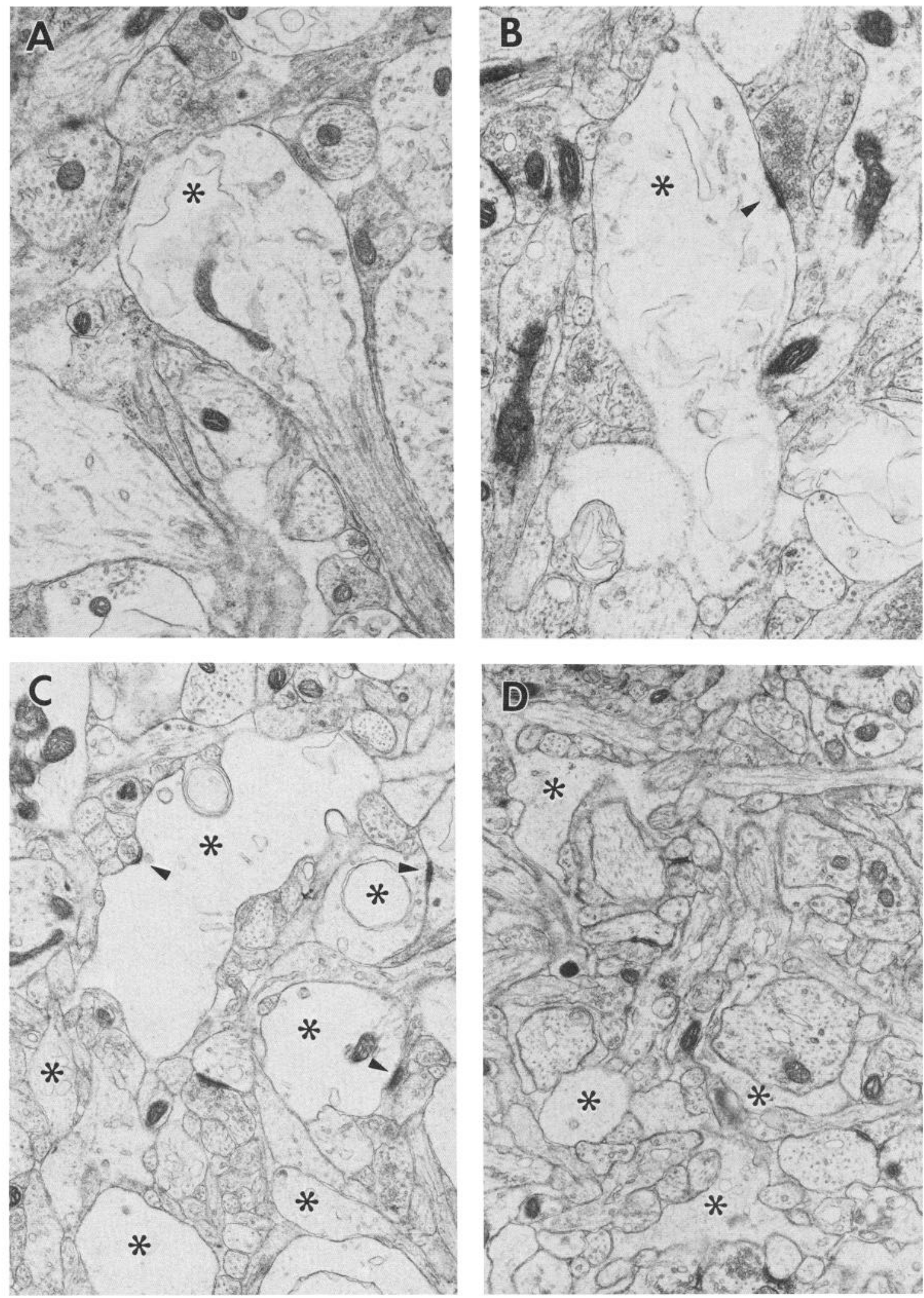

Figure 1. Growth cones in the developing tree shrew dLGN. These electron micrographs illustrate the criteria used to classify developing processes as growth cones $\left(^{*}\right)$ : (1) a minimum of $0.5 \mu \mathrm{m}^{2}$ in area, (2) filled with a microfilamentous feltwork, (3) no more than 1 neurotubule in leading edge of growth cone enlargement, (4) no more than 3 rosettes of polyribosomes, (5) at least one membranous sac, (6) no organelles other than mitochondria and various types of sacs. $A$, P0: Typical bulbous growth cone. $\times 22,400 . B$, P4: Large, bulbous, postsynaptic growth cone with synapse at arrowhead. $\times 22,400$. C , P8: Note the variety in growth cone appearance; some are bulbous, while others are smaller and denser. Arrowheads indicate synaptic densities. $\times 16,800 . D$, P14: Growth cones at this age are generally much more linear in shape. $\times 16,800$. 


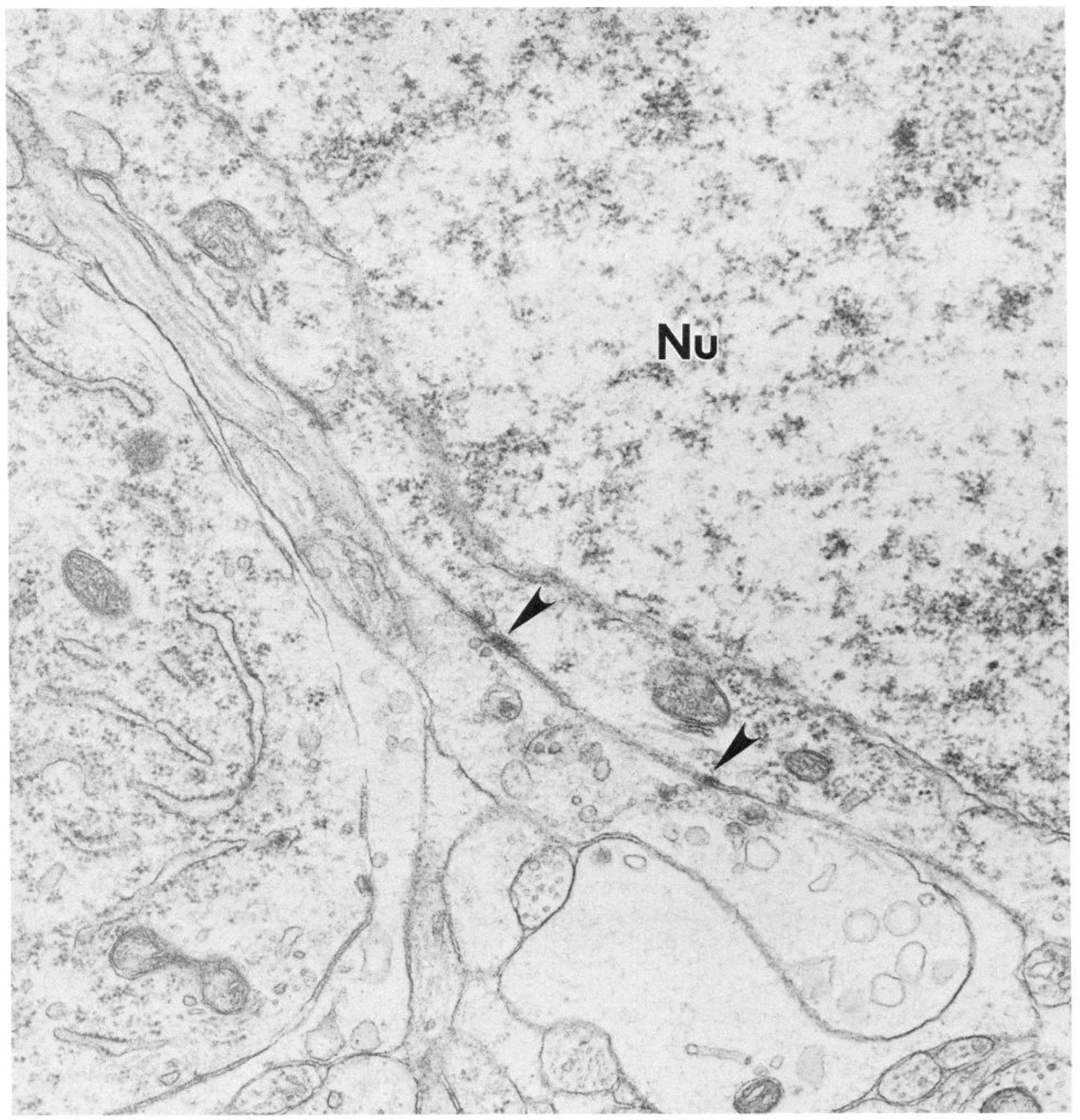

Figure 2. Presynaptic growth cone at P8. This axon profile expands into a bulbous growth cone swelling. Arrowheads indicate 2 developing somatic synapses. $N u$, nucleus. $\times 36,000$.

a microfilamentous feltwork, had no more than one neurotubule in the leading edge of the growth cone enlargement, had no more than 3 rosettes of polyribosomes, had at least one membranous sac, and had no organelles other than mitochondria and various types of vesicles. Profiles meeting these criteria ranged from large bulbous structures to small dense structures (Fig. 1).

The identification of a growth cone as axonal or dendritic is quite difficult. Shape is not a useful criterion; bulbous growth cones can either be dendritic, as illustrated by the postsynaptic growth cones in Figure $1 B, C$ (arrowheads), or axonal, as illustrated by the presynaptic growth cone in Figure 2 . In the present study, growth cones were classified as pre- or postsynaptic only if an obvious synaptic profile was present. However, as only $10 \%$ of the growth cones could be identified as clearly pre- or postsynaptic according to our criteria, these categories were not included in the statistical analyses.

Quantitative data. An overview of changes in growth cone number during development is illustrated in Figure $3 A$. This graph presents the mean number of growth cones in $10,000 \mu \mathrm{m}^{2}$ of neuropil at each age studied. With increasing age, there is a significant decrease in the mean number of dLGN growth cones $(p=0.012)$. At $\mathrm{P} 15$, there is a transient elevation in growth cone number in comparison with P7 and P90. Although we cannot rule out a real increase in growth cone number at this stage of development, it may be that this elevation is due to an observed increase in developing dendritic spines, which are similar in size and appearance to growth cones at this age. Also, during these early stages of rapid brain development, we cannot 

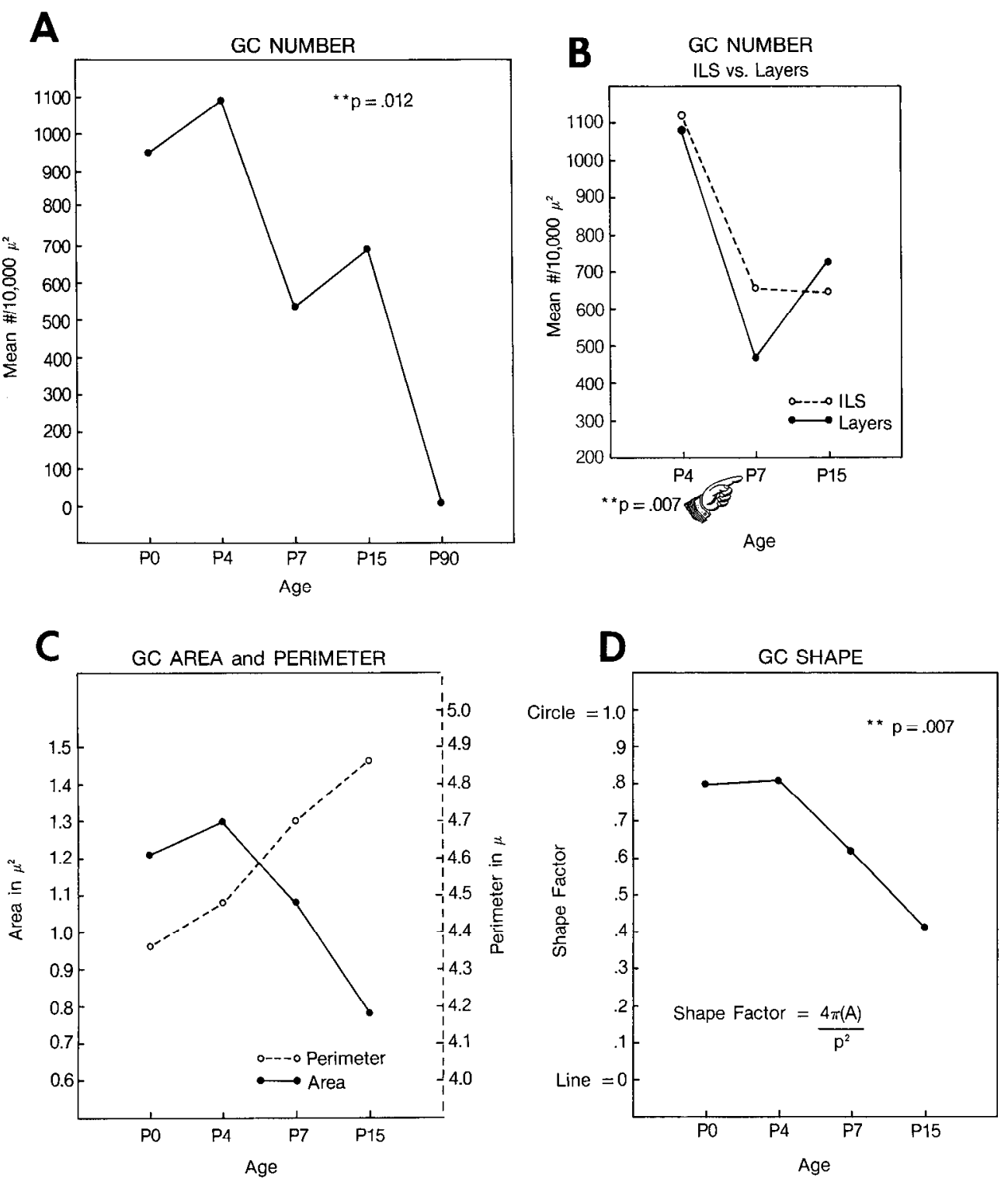

Figure 3. Quantitative growth cone data. $A$, Mean number of growth cones in $10,000 \mu \mathrm{m}^{2}$ at each age studied. A one-way analysis of variance indicates a significance difference in mean growth cone number between the various ages $(p=0.012)$. The transient increase in growth cone number at P15 may be due to inclusion of some spines in our counts; immature spines can be quite large and can share features of growth cones. $B$, Mean number of growth cones per $10,000 \mu \mathrm{m}^{2}$ in the interlaminar space and layers at each age in which both growth cones and interlaminar space are present (P4, P7, and P15). The only significant difference is at $\mathrm{P} 7(=0.007)$. At P7 the interlaminar space between layers 4 and 5 has been present for approximately $5 \mathrm{~d}$, suggesting that the greater number of growth cones in the interlaminar space may be a response to the laminar segregation rather than a causal factor. $C$, Growth cone area and perimeter at each age studied. There is a slight increase in growth cone area between $\mathrm{P} 0$ and $\mathrm{P} 4$; thereafter there is a continuous decrease in area with age. In contrast, there is a steady increase in growth cone perimenter at each age studied. $D$, Growth cone shape at each stage studied. The data illustrated in Figure 4 were used to quantify growth cone shape (shape factor $4 \pi(A) / p^{2}$ ). A circle has a shape factor of 1.0 and a line has one of 0 . After P4, dLGN growth cones gradually become less rounded. rule out the possibility that some of the growth cones may be glial in origin.

A comparison between the number of growth cones in the layers and the interlaminar space is illustrated in the graph in Figure $3 B$. Only $\mathrm{P} 4, \mathrm{P} 7$, and $\mathrm{P} 15$ are included here because interlaminar spaces are not present at $\mathrm{P} 0$ and growth cones are not present at $P 90$. Statistical analysis shows a significant overall effect of location with age $(p=0.022)$. Subsequent paired $t$ tests at each age however, indicate that these is no significant difference between laminar and interlaminar values at P4 $(p=0.235)$ or P15 ( $p-0.346)$; the only significant difference between laminar and interlaminar values occurs at P7 $(p=0.007)$, nearly a week after laminar segregation has begun.

One of the most readily apparent changes in growth cones during the period of interlaminar space formation is the change in size and shape. This difference is illustrated in the electron micrographs in Figure 4, in which growth cones are indicated by asterisks. In Figure $4 A$, numerous large, spherical growth cones can be seen; this example is from $\mathrm{P} 4$ but is quite typical of the appearance of many parts of the neuropil at P0 as well. In marked contrast, Figure $4 B$ illustrates the neuropil from the
dLGN of a tree shrew sacrificed on P15, in which the growth cones are much smaller in area and more linear in shape.

To determine whether this change in shape is significant, we measured the areas and perimeters of all growth cones in our sample. We used these data to determine a shape factor for each growth cone using the formula $4 \pi(A) / p^{2}$, where $A$ is area and $p$, perimeter. In this analysis, a value of 1.0 is a perfect circle and values approaching 0 approach a straight line. Although growth cones tended to be somewhat larger in the interlaminar space than in the layers, there are no statistically significant interactions between layers and the interlaminar space in the perimeter, area, or shape factor of the growth cones. Therefore, raw data from the layers and interlaminar space were combined, corrected for area, and subsequently analyzed for the effect of age.

The combined perimeter and area data are shown in Figure $3 C$. During the first 2 weeks of postnatal development in the tree shrew dLGN, growth cone perimeter increases steadily. During that same period, growth cone area increases slightly to a peak at P4 and then decreases steadily. The changes in shape factor with age are shown in Figure $3 D$. During the first half of 

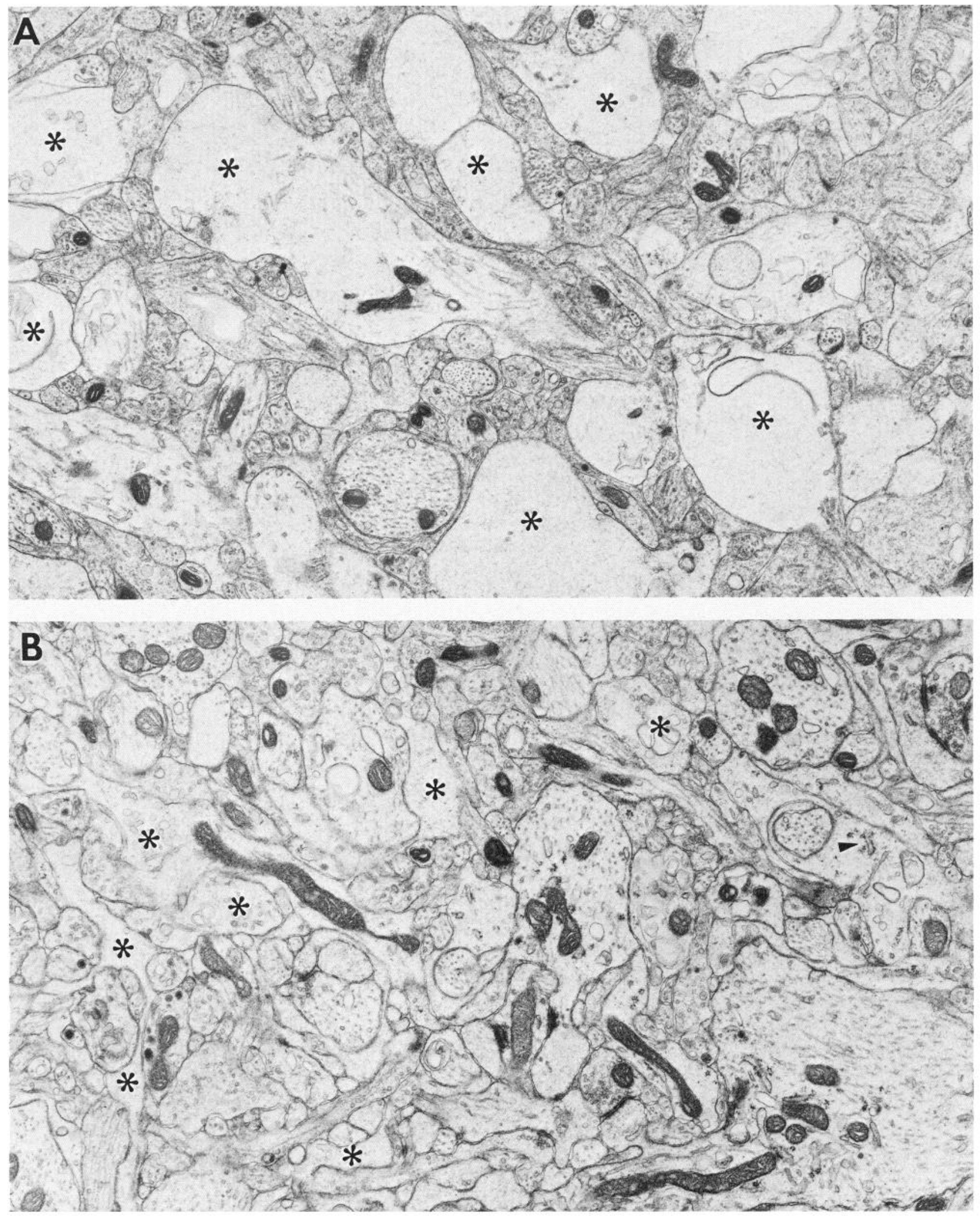

Figure 4. Comparison of typical fields of growth cones at P4 and P15. Growth cones are indicated by asterisks at P4 $(A)$ and P15 $(B)$. There is a considerable change in shape between these ages as the profiles become less bulbous and more linear. Arrowhead in $B$ indicates small amount of rough endoplasmic reticulum in a profile that would result in its not being counted as a growth cone in our sample. $\times 18,000$. 
the first postnatal week ( $\mathrm{P} 0$ and $\mathrm{P} 4)$, the growth cones are quite circular (shape factor $=0.8$ ), whereas by the end of the second postnatal week (P15), they are considerably more linear (shape factor - 0.4). An analysis of variance indicates this change in shape factor with age is statistically significant $(p=0.007)$.

\section{Synapses}

Morphology. In this portion of our study, our main objective was to determine whether there is a significant difference in synaptic density at any age between the layers and interlaminar spaces, not to study the development of differences in synaptic types. Nevertheless, we were faced with the special problem of establishing classifications that reflect the nonhomogeneity of dLGN synaptic profiles but that also are useful at very early stages of development when the characteristics of mature dLGN synapses (Guillery, 1969) cannot yet be recognized. Accordingly, we have classified terminals as either "simple terminals" (STs), i.e., those having a single contact, or as "multiple terminals" (MTs), i.e., those having more than one synaptic contact. In all instances, the criteria for a synapse were the presence of at least 3 synaptic vesicles in the vicinity of the synaptic site and the presence of a clear postsynaptic thickening.

Figure 5 illustrates synapses in the adult (P90) dLGN for comparison with those in the developing dLGN. The synapses illustrated in Figure 5, $A$ and $B$, are classified as STs in the present study. The round vesicles in these small, dense terminals suggest that these profiles correspond to the RSD profiles of Guillery in cats (1969) and to the RS profiles of Hajdu et al. (1982) in tree shrews, which have been demonstrated to be primarily extraretinal in origin (Szentágothai et al., 1966; Jones and Powell, 1969; Hajdu et al., 1982). Nevertheless, some small, simple terminals in the cat dLGN have been demonstrated also to be retinal in origin (Robson and Mason, 1979). Figure 5, C and $D$, illustrates examples of large MTs. The round vesicles and large, pale mitochondria, as well as the glial encapsulations (arrowheads in Fig. 5C) suggest that these profiles correspond to the RLP profiles in cats (Guillery, 1969) and to the RL profiles in tree shrews (Hajdu et al., 1982); similar profiles have been described in other species as well (e.g., Colonnier and Guillery, 1964). These complex terminals are part of the synaptic glomeruli characteristic of the mature dLGN (Szentágothai, 1970) and are known to be retinal in origin (Szentágothai et al., 1966; Guillery, 1969; LeVay, 1971; Wong-Riley, 1972b; Hajdu et al., 1982). In addition to the RSD and RLP profiles, F-type synapses containing flattened or pleiomorphic vesicles (Fig. 5, $C, D$ ) are evident in the adult and are associated with intrinsic connections (Szentágothai, 1963; Guillery, 1969; Wong-Riley, 1972a; Hajdu et al., 1982). While RSDs would belong almost exclusively to our ST category in the adult (Guillery, 1966) and the RLPs would most commonly belong to the MT category, it must be emphasized that there is no direct equivalence between the catcgorics, nor did we intend to establish one. Our goal was quite simply to establish a very basic categorization that would be useful in quantifying synaptogenesis at all developmental stages.

Many of the features described above that distinguish mature synaptic types are not present in the immature tree shrew dLGN. In fact, although STs are present at P0 (Fig. 6, $A, B$ ), MTs are rarely seen. By P4, STs are more common (Fig. 6D), and MTs (Fig. 6C) are beginning to be seen as well. At P4, however, glial encapsulations are not present, and other defining characteristics are absent as well.

The earliest examples of terminals containing flattened ves- icles are illustrated at $\mathrm{P} 7$ in Figure $7, B-D$. In addition, Figure $7 A$ illustrates a typical example of an ST (arrowhead) synapsing on a large growth cone at P7. Spinous synapses, although occasionally present at $\mathrm{P0}$ and $\mathrm{P} 4$, are considerably more frequent by P7; MTs synapsing on several spines are shown in Figure 7C. Although MTs are more common at $\mathrm{P} 7$, many are quite simple in appearance (Fig. $7 D$ ).

At P15, synapses often terminate on spines (S) (Fig. 8), some of which are connected to the dendritic shaft by long necks (Fig. $8 A$ ). At this stage, synapses on dendritic shafts are also common and are illustrated in Figure $8, A, B$. By $\mathrm{P} 15$, the neuropil is becoming more mature in appearance: MTs at this stage of development are abundant (Fig. 8, C, D) and are more likely to be encapsulated (arrowhead, Fig. $8 C$ ) and to be found in more complex synaptic configurations, as exemplified by the F-type contact in Figure $8 D$.

Quantitative data. The postnatal changes in total number of synapsing STs and MTs per $10,000 \mu \mathrm{m}^{2}$ of neuropil are shown in Figure $9 \mathrm{~A}$. Clearly, throughout development, STs are more numerous than MTs. Both STs and MTs increase gradually between P0 and P7. There is a large increase in the number of STs by P15, and this increase continues between P15 and P90; at P90 the number of STs is nearly double that at P7. The number of MTs also increases somewhat between P7 and P15, but then decreases by P90. An analysis of variance indicates a significant difference with age in the mean number of synapsing terminals of both types (ST, $p=0.001 ; \mathrm{MT}, p=0.008$ ).

The postnatal changes in total number of synaptic contacts per $10,000 \mu \mathrm{m}^{2}$ of neuropil (i.e., number per area) in the layers and interlaminar space, irrespective of terminal type, are shown in Figure $9 B$. While there are always more synapses in the layers than in the interlaminar space, $t$ tests indicate no significant difference at any particular age. The cumulative effect of consistently greater synapse number in layers versus interlaminal space is significant $(p=0.008)$, however, as is the effect of age $(p=0.003)$. To be certain that there were no differences masked by variation in the size of synaptic elements in the layers and interlaminar spaces, we measured the mean lengths of the synaptic densities and subsequently derived a number per volume for the interlaminar space and the layers using the formula $N V=$ $N A /(T+M L)$, where $N V$ is the number per volume; $N A$, the number per area; $T$, section thickness; and ML, mean synaptic length. These data revealed $p$ values equivalent to those based on number per area. In summary, synaptogenesis increases at roughly the same rate in both regions throughout development, and there is no significant difference in synapse number with location at any age.

To analyze the distribution of postnatal synaptogenesis more closely, we compared the percentage of synapsing terminals in the interlaminar space that were STs or MTs with the percentage of synapsing terminals in the layers that were STs or MTs. This comparison is illustrated in Figure $10 \mathrm{~A}$. Although STs are always more common in the interlaminar space than in the layers, they are clearly the dominant synaptic type in both locations, and there is no significant effect of ST location with age. On the other hand, there are more MTs in the layers than in the interlaminar space, and statistical analysis revealed that the overall effect of the location of those terminals with age was significant $(p=0.050)$. In addition, the percentage of laminar MTs increases between $\mathrm{P} 4$ and $\mathrm{P} 7$, which may reflect a maturation of laminar STs into MTs.

By also identifying the postsynaptic element, we have been 

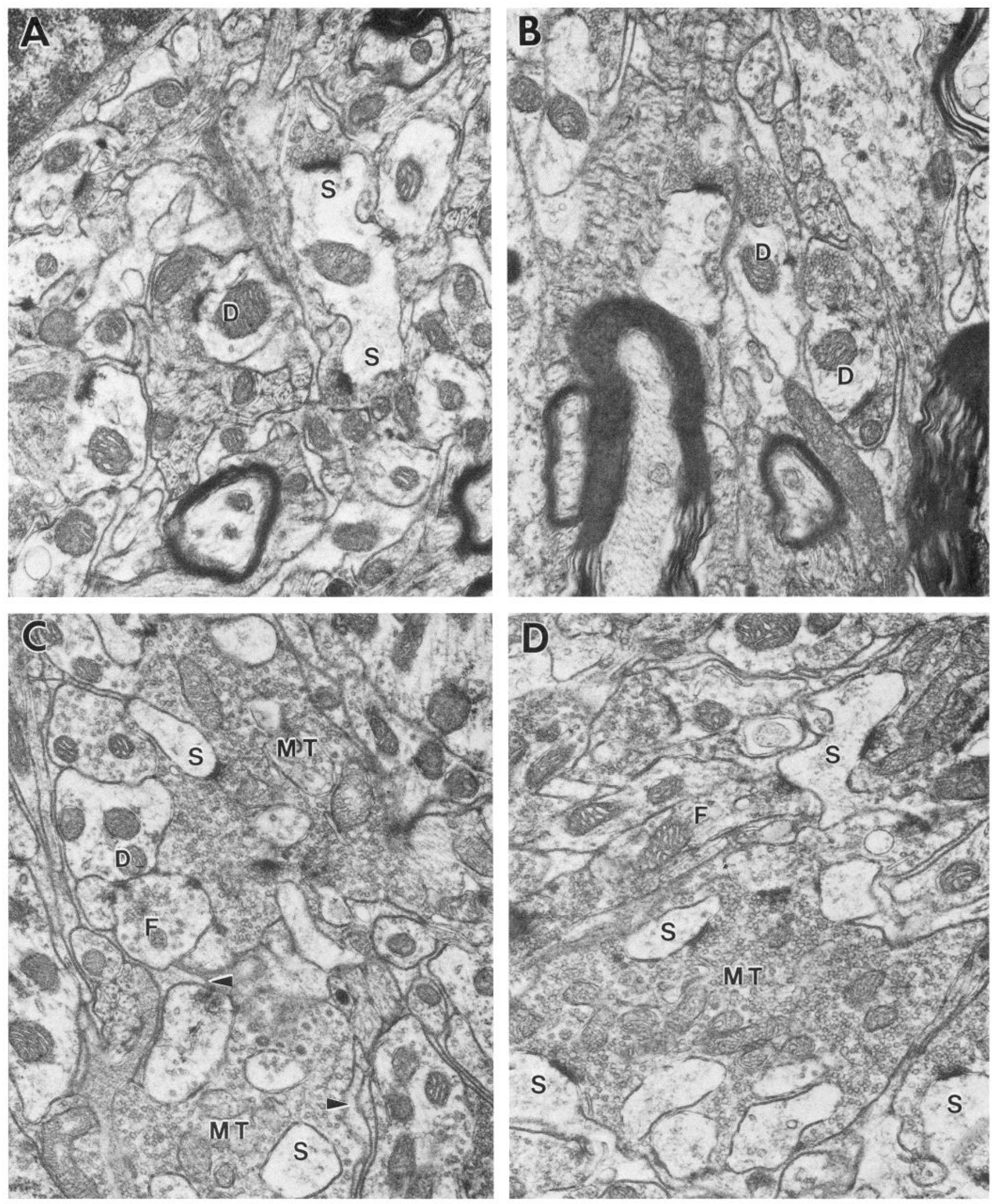

Figure 5. Synapses in the tree shrew dLGN at P90 (maturity). $A$ and $B$ illustrate profiles which would be counted as STs in the present study. They are comparable to the RSD terminals of Guillery (1969) and RS terminals of Hajdu et al. (1982). $C$ and $D$ illustrate MTs which are comparable to the RLP terminals of Guillery (1969) and RL terminals of Hajdu (1982). F-type profiles can be seen as well. Arrowheads point out glial encapsulations: $S$, dendritic spine; $D$, dendritic shaft; $M T$, multiple terminal; $F$, type $\mathrm{F}$ synapse. $\times 21,500$. 

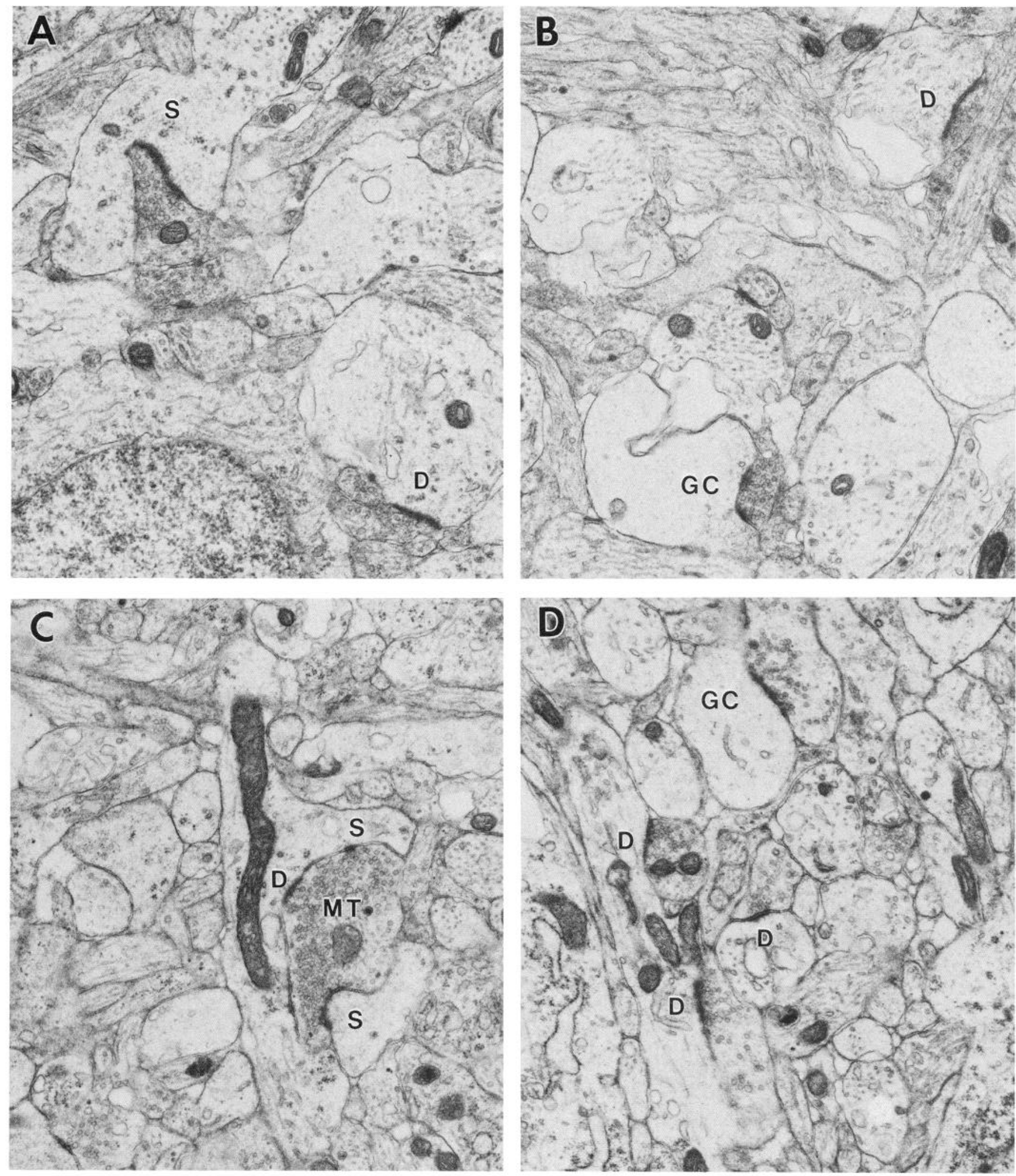

Figure 6. Synapses in the tree shrew dLGN at P0 and P4. $A$ and $B$, Illustrate STs at P0, $C, M T$ at P4; $D$, STs at P4. MTs are infrequently encountered at this age. $S$, dendritic spine; $D$, dendritic shaft; $G C$, growth cone; $M T$, multiple terminal. $\times 21,500$.

able to compare the mean number per area of spinous and dendritic synapses per $10,000 \mu \mathrm{m}^{2}$ of neuropil during postnatal development; these data are shown in Figure 10B. Analysis of the data reveals a significant interaction between synaptic type and age $(p=0.008)$, as well as significant main effects of age ( $p$ $=0.0003)$ and synaptic type $(p=0.0006)$. Paired $t$ tests to examine the difference at each age, however, show significance only at P15 $(p=0.04)$. At both P0 and P90, the number of 

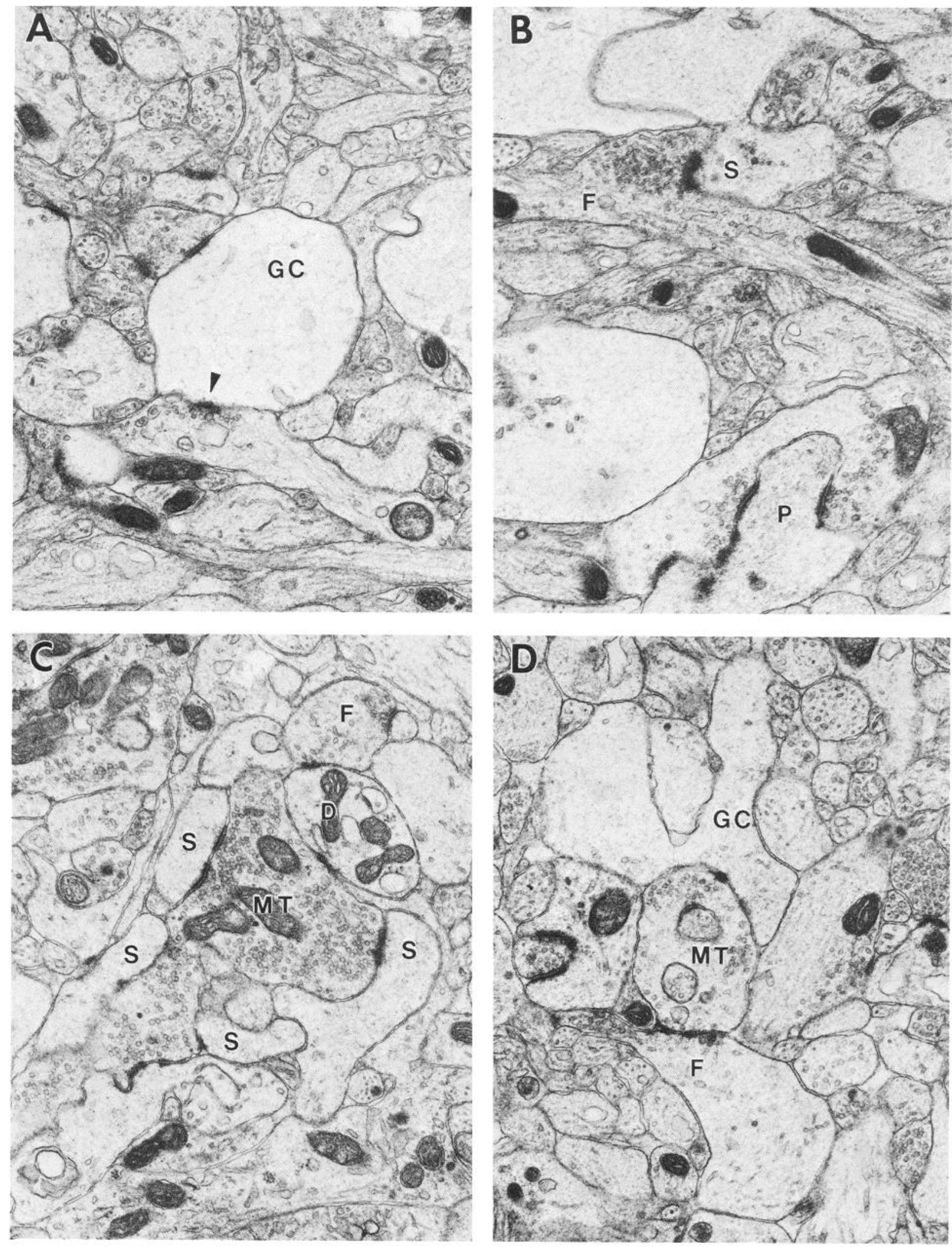

Figure 7. Synapses in the tree shrew dLGN at P7. A, Arrowhead indicates an ST synapsing on a large growth cone. B, A perforated synapse and one of the earliest examples of an F-type synapse. $C$, A large MT synapsing on several spines and a smaller MT containing pleiomorphic vesicles. $D$, MT in contact with a growth cone and a F-type profile. $P$, perforated synapse; $F$, type F synapse; $M T$, multiple terminal; $G C$, growth cone; $S$, dendritic spine; $D$, dendritic shaft. $\times 21,500$. 

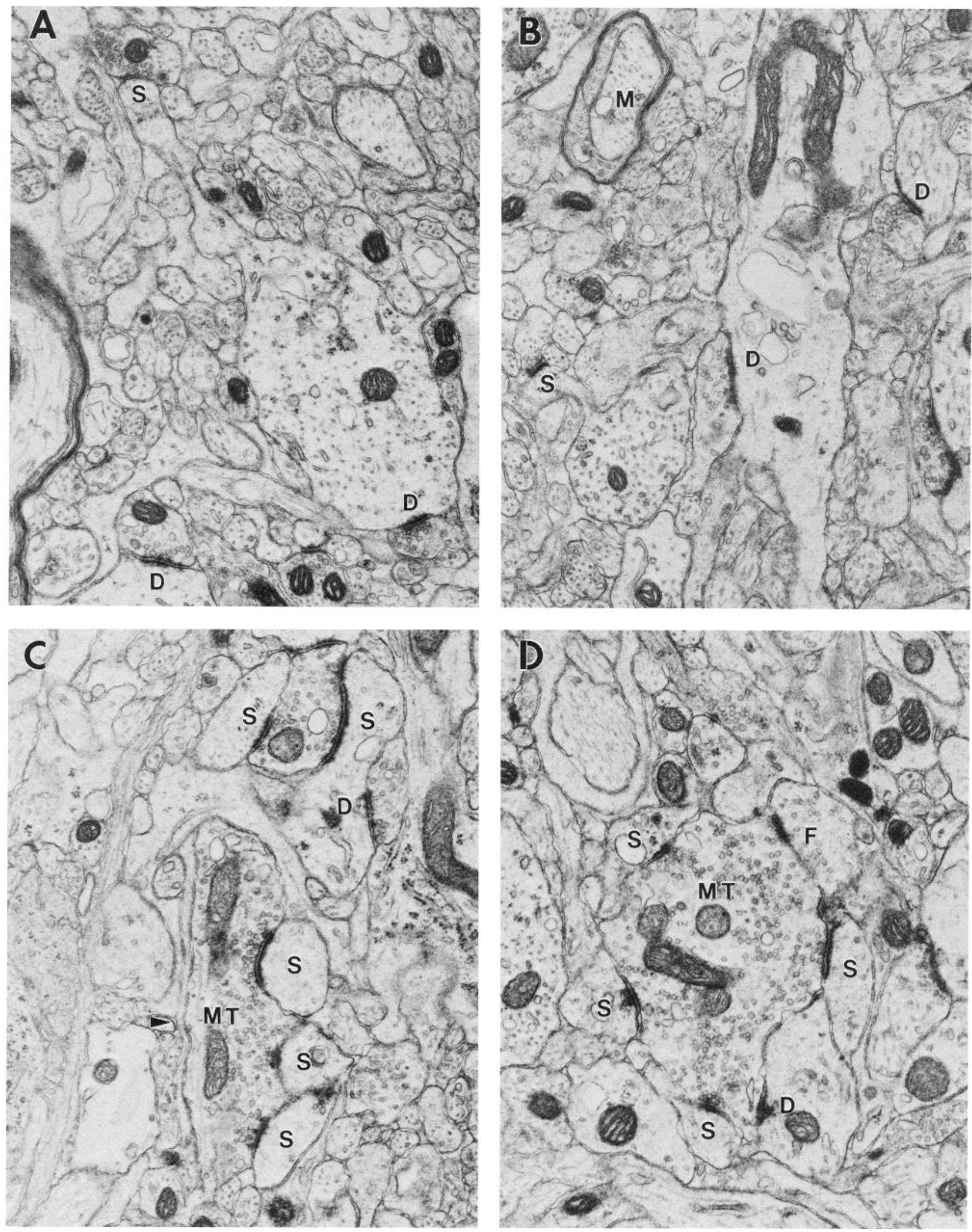

Figure 8. Synapses in the tree shrew dLGN at P15. A, Note the long neck of the simple spinous contact. $B$, Synapses on dendritic shafts as well as on a small spine connected to a dendrite. $C$, MT contacting numerous dendritic spines; arrowhead indicates glial encapsulation. $D$, Another MT showing the beginning of more complex synaptic configurations. $D$, dendritic shaft; $S$, dendritic spine; $M$, axon undergoing myelination; $M T$, multiple terminal. $\times 23,400$. 
Figure 9. A, Mean number of synapsing multiple and simple dLGN terminals in $10,000 \mu \mathrm{m}^{2}$ at each age studied. At all ages there are more STs than MTs. The number of STs increases throughout postnatal development $(p=0.001)$ with the largest increase between $P 7$ and $P 15$. There is also a significant interaction between number of MTs and age $(p=0.008)$. The number of MTs gradually increases to a peak at P15 after which it slightly decreases. $B$, Mean number of synapses per $10,000 \mu \mathrm{m}^{2}$ in interlaminar space and layers at each age studied after the beginning of laminar segregation. The number of synaptic contacts in both the layers and interlaminar space increases throughout postnatal development. Paired $t$ tests at each age indicate there is no significant difference in synapse number between the layers and interlaminar space at any age, suggesting that synaptogenesis at the dLGN laminar borders is unlikely to be the causal factor initiating cell layer segregation.
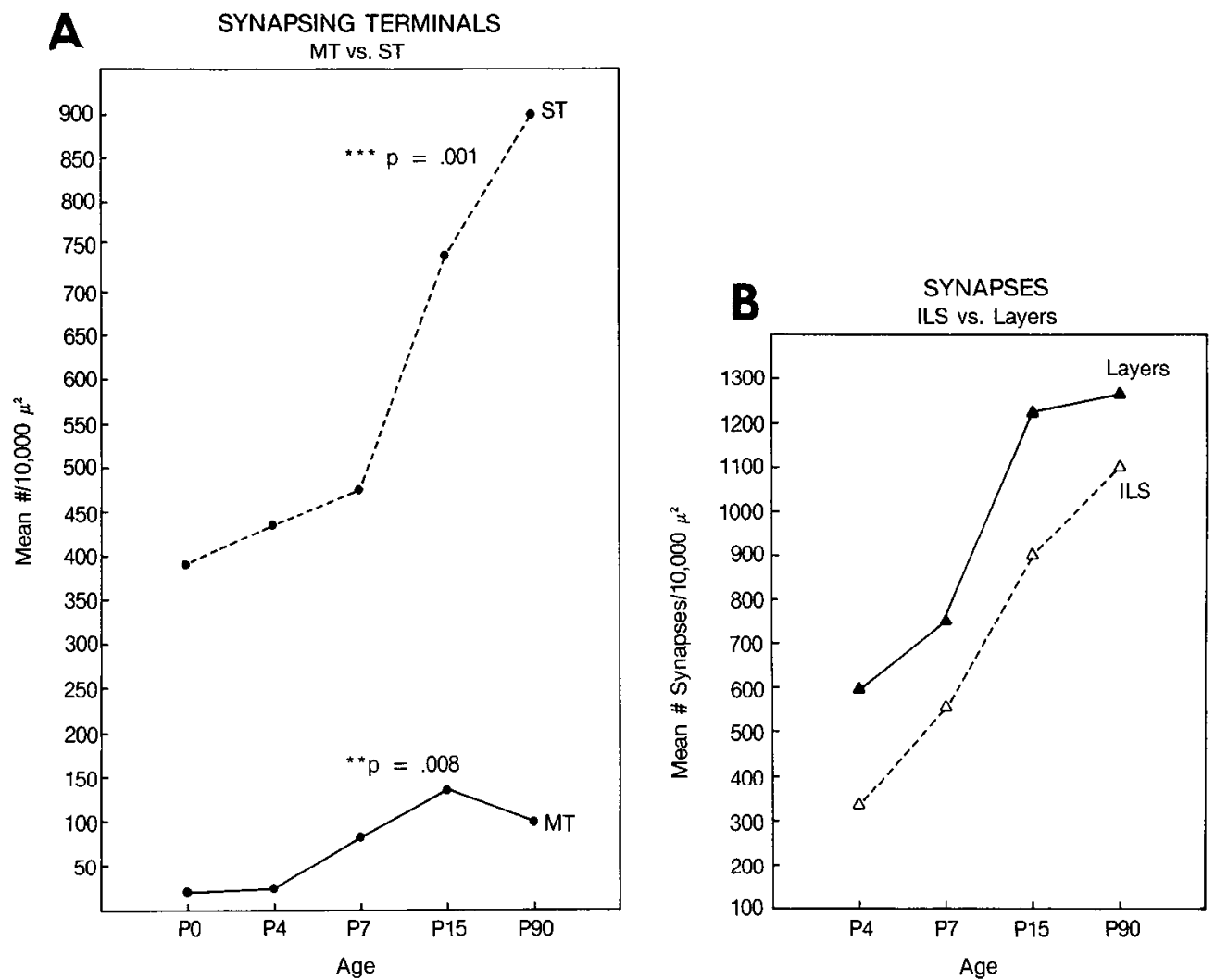

spinous and dendritic synapses is basically equivalent. During the intervening period, spinous synapses increase to a peak at P15, after which they decline. Concomitantly, dendritic synapses remain low until P15, after which they increase. These observations are consistent with a decrease in the number of dendritic spines between P15 and maturity.

\section{Discussion}

\section{Comparison of layers 4 and 5}

Although data were collected separately for layers 4 and 5 , there were no significant differences between the 2 layers. Studies in other species have suggested that the development of the contralateral retinogeniculate projections is in advance of the ipsilateral one (Lund and Bunt, 1976; So et al., 1978; Linden et al., 1981; Shatz, 1983). That this is not reflected in our data may be due to one or more of several factors. First, a temporal difference in development between the ipsilateral and contralateral retinogeniculate projections may not be present in tree shrews. Second, since our data are collected well after the segregation of the ipsilateral and contralateral retinogeniculate projections, maturational differences between these innervated layers may have equilibrated. Finally, contralateral and ipsilateral differences in the retinogeniculate projection may not be reflected in growth cone or synaptic density.

\section{Growth cones}

The present results provide qualitative as well as quantitative information on the nature of the neuropil in the dLGN during the period of cell layer segregation. Detailed information is available in the literature on the development of individual retinogeniculate fibers (e.g., Robson and Mason, 1979; Mason, 1982a, b; Sretavan and Shatz, 1984; Sretavan and Shatz, 1986); however, little data has been published on the changing mor- phology of growth cones and synapses in the dLGN at the time cell layers are forming.

The appearance of the profiles classified as growth cones in our study is consistent with many descriptions of growth cones in the literalure, based on in vitro (Bunge, 1973; Yamada et al., 1974), as well as in vivo studies (Del Cerro and Snider, 1968; Tennyson, 1970; Hinds and Hinds, 1972; Skoff and Hamburger, 1974; Vaughn et al., 1974; Williams et al., 1986). In all descriptive studies, growth cones are reported to contain a microfilamentous feltwork and membranous vesicles with relatively few organelles. These vesicles are particularly abundant in in vitro studies (e.g., Bunge, 1973). In the present study, we often see large growth cone profiles filled with microfilamentous feltwork and containing only 1 or 2 small vesicular sacs. The overall similarity between the appearance of growth cones in the present study and those in the literature (see above), as well as the wellfixed appearance of membranes and other structures such as mitochondria, suggests that the often bulbous appearance of the growth cone profiles is a valid feature of their morphology rather than the result of poor tissue preservation.

Although several studies of growth cones have addressed the issue of axonal versus dendritic growth cone morphology, it is difficult to determine growth cone identity unequivocally. For example, studies addressing in vitro (Pfenninger and Bunge, 1974) and reactive growth cone formation (Knyihár-Csillik et al., 1985) may identify characteristics that differ from developing axonal and dendritic growth cone profiles in vivo. Serial reconstructions in vivo in embryonic tissue have shed some light on characteristics of dendritic (Hinds and Hinds, 1972; Skoff and Hamburger, 1974; Vaughn et al., 1974) and axonal growth cones (Skoff and Hamburger, 1974). In one of these studies, dendritic growth cones are described as regular and either bulbous with sparse organelles or rather small and dense, whereas all axonal 


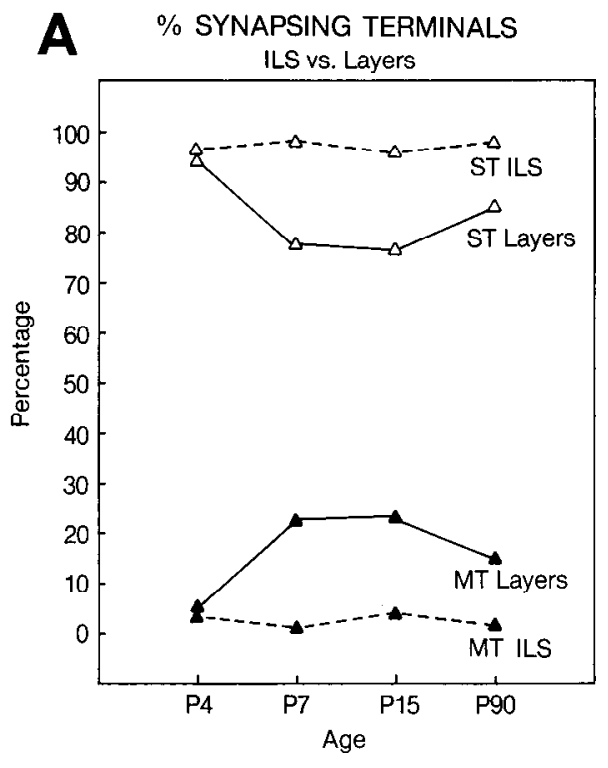

Figure 10. A, Percent synapsing simple and multiple terminals in the interlaminar space and layers at each age studied after the beginning of laminar segregation. STs make up a larger percentage of synapsing terminals in both the layers and the interlaminar space. The increase, however, between P4 and P7 in the percentage of laminar MTs may reflect the maturation of some STs into MTs. $B$, Mean number of spinous and dendritic synapses in $10,000 \mu \mathrm{m}^{2}$ at each age studied. At P4, P7, and P15 during dLGN development there are more synapses on spines than on dendrites. Between P15 and P90, the mean number of spinous synapses decreases and the mean number of dendritic synapses increases to roughly comparable levels. Statistical analysis reveals a significant interaction between synapse type and age $(p=0.008)$; however, paired $t$ tests reveal the only age at which the difference between synapse type is significantly different is at P15 $(p=$ $0.04)$. The changes in synapse type with age may be correlated with decreases in the number of dendritic spines between early development and maturity. growth cone profiles are described as dense but with a more irregular profile (Skoff and Hamburger, 1974).

In the tree shrew dLGN the size and shape of individual growth cones appear to be directly related to the stage of development, i.e., with increasing age the growth cones become increasingly linear. This observation may be due to one or both of 2 factors. First, both axonal and dendritic growth cones may become more linear either as they simply become more mature and send out increasingly finer processes or as a response to the increasing mass or changing nature of the neuropil. This is consistent with the idea that growth cones may change shape during development in response to changes in the extracellular environment (Norlander and Singer, 1982; Mason, 1985a, b). It may also be that the shape of growth cones may vary depending on the role they are playing in relation to other growth cones of the same type (Reh and Constantine-Paton, 1985). Second, it may be that only organelle-sparse, dendritic growth cones with a bulbous morphology are present early in development and that these are then replaced by denser, more linear dendritic, as well as axonal, growth cones.

While it is impossible to distinguish between these factors without serial reconstructions at each of the ages in our study, several relevant observations can be made. For example, we often see bulbous profiles that are clearly presynaptic. Although the presence of presynaptic dendrites in the mature dLGN suggests that it is possible that the presynaptic growth cones in the early postnatal dLGN are dendritic, the overall immaturity of synaptic configurations at that stage of development makes it unlikely that all bulbous presynaptic growth cones are dendritic in origin. In addition, light microscopic evidence has demonstrated that the retinal fibers are not only in the dLGN at birth in tree shrews but are also segregated into eye-specific bands (Brunso-Bechtold and Casagrande, 1982). Since bulbous growth cones are by far the predominant morphological type at birth, it is quite likely that at least some of them are axonal. Thus, while dendritic and axonal growth cones undoubtedly vary in morphology, the maturation of the growth cone and its relation to the surrounding neuropil also may play a significant role in the determination of growth cone shape and size.

It is perhaps not surprising that there is a decrease in growth cone number after P4. By that time retinal input is well established (Brunso-Bechtold and Casagrande, 1982), input from the superior colliculus is present, and input from the visual cortex has begun to arrive (Brunso-Bechtold and Casagrande, 1984). Between P7 and P15, however, there is an increase in growth cone number to a transient peak at P15. While there may be an actual transient, secondary increase in growth cone number, it may well be that the peak is instead due to a proliferation of dendritic spines at that stage of development. Several points support this conclusion. First, qualitative observation reveals a massive development of dendritic spines at P15; we frequently observe spines with long necks and large collections of polyribosomes located at their bases in the dendritic shaft (see Steward and Falk, 1986). Second, our quantitative data reveal that growth cone areas are decreased to an average of $0.75 \mu \mathrm{m}^{2}$ at P15. This puts many of the growth cones in the size range of large developing spines. Since developing spines share many characteristics with growth cones, (i.e., presence of filamentous feltwork, paucity of organelles), some may have been unavoidably included in our sample.

The segregation of cells into individual layers begins about P2 in tree shrews. By P4 the interlaminar space between layers 4 and 5 is wide enough to be clearly distinguishable. At that stage of development, there is no significant difference between the number of growth cones or size of growth cones in the layers and interlaminar spaces. By $\mathrm{P} 7$, however, well after the interlaminar space between layers 4 and 5 can be distinguished, there are significantly more growth cones in the interlaminar space than in the adjacent layers. Since this difference is not present at $\mathrm{P} 4$, it is likely that, rather than playing a role in initiating the 
segregation of cell layers, the growth cones may instead grow into the already formed interlaminar space, thus playing a role in widening them.

\section{Synapses}

It should be reemphasized here that the goal of the present study was to determine whether synaptogenesis plays a role in initiating the segregation of dLGN cells into layers. Thus, the primary concern was one of quantification, not one of categorization. However, it also seemed important to quantify the results in a manner that reflected the lack of homogeneity of synaptic type apparent even before mature synaptic classifications can be distinguished. Consequently, synapses were categorized as STs and MTs, depending on whether the terminal had one or more synaptic contacts on different postsynaptic elements.

At P0, all synapses fall in the category of STs. By P4, a few MTs are present, but the pale, watery mitochondria and glial encapsulations characteristic of RLPs cannot yet be distinguished. It is not until P7 that hints of the adult synaptic morphology can first be distinguished. At that time, a few F-type terminals containing flattened vesicles and some presynaptic dendrites are present. Fven so, these features are not common enough to be used as the basis of a meaningful categorization. By P15 the synaptic profiles are beginning to take on a more mature appearance and the beginnings of synaptic terminal encapsulations can be seen, but the quantification of most terminals would still be equivocal using criteria established in the adult.

One issue regarding synaptic type does arise, however, concerning the synaptic terminals present at P0. At that age no MT profiles are present. At P0, however, retinogeniculate fibers are known to be segregated into eye-specific bands in tree shrew dLGN (Brunso-Bechtold and Casagrande, 1982). At an even earlier developmental stage in cats, retinogeniculate fibers have been demonstrated to make functional contacts (Shatz and Kirkwood, 1984). In addition, in the P0 tree shrew, light microscopic results suggest that the majority of the corticogeniculate projection have not yet entered the nucleus and that the colliculogeniculate projection is not yet mature (Brunso-Bechtold and Casagrande, 1984). It thus seems unlikely that all of the ST profiles present at P0 are extraretinal in origin. A more likely possibility is that many of the STs present at P0 are instead immature retinal terminals, which eventually mature into large, complex MTs. This possibility is further supported by the observation that the percentage of MTs in the layers increases approximately 2 -fold between P4 and P7. Since the predominant site of retinogeniculate termination is the layers, this suggests that some of the laminar STs may be maturing into MTs between $\mathrm{P} 4$ and $\mathrm{P} 7$.

Regarding the distribution of postsynaptic elements (i.e., spine, dendrite, growth cone), there is no significant effect of location (laycr vs. intcrlaminar space) or tcrminal type (ST vs. MT). There is, however, a significant difference in the overall numbers of spinous versus dendritic synapses. As previously discussed, at $\mathrm{PO}$ the numbers of synapses on spines and dendrites are roughly equivalent, as they are at maturity. However, during the first 2 postnatal weeks there is a much greater increase in the number of synapses on spines than on dendrites, with the number of spinous synapses reaching a peak at P15. This is the same age at which we have suggested that our transient peak in growth cone number may, in fact, be due to the inclusion of developing spines in our sample. A similar transient period of numerous dendritic spines has been demonstrated in numerous systems, including the developing auditory hindbrain (Smith, 1981) and the visual cortex (Boothe et al., 1979; Michel and Garey, 1984). In addition, Sretavan and Shatz (1986) used anterograde filling of retinogeniculate fibers with HRP to demonstrate that at early stages of development those fibers are covered with fine side branches. Although comparison of the times of laminar segregation in the 2 species suggests that that period in cats is considerably earlier in development than the peak of dendritic spines in tree shrews, it is difficult to make direct temporal comparisons between the species. It would be interesting to know whether there is any relation between the period of transient axonal side branches observed in cats and the period of transient dendritic spines we see in tree shrews. The reduction of dendritic spines as development progresses also could be explained by the absorption of the spine neck once permanent contacts are in place in order to conserve space as myelination increases.

The basic picture presented by our results is one of steadily increasing synapse formation during postnatal development. This increase can be seen in the number of synapses in both the layers and the interlaminar space. Although there are always more synapses present in the layers, and there is a cumulative main effect of location ( $p=0.008)$, there is no significant difference in synaptic number with location at any particular age. This steady increase in synapse number during dLGN development is in contrast to the observation in many systems that there is an overproduction of synapses during development and a subsequent decrease at maturity (see Hopkins and Brown, 1984). It is further curious in light of the transient peak in dendritic spines suggested by our results; dendritic spine development is believed to mirror the ongoing synapse formation (e.g., Morest, 1969; Hamori, 1973). It may well be that there is a transient peak in synapse density between P15 and maturity that we have missed due to a lack of time points in that interval. Nevertheless, the lack of any peak in synapse number during laminar segregation, coupled with the fact that synapses are more prevalent in the layers than the interlaminar space, argues against the role of differential synapse formation along laminar borders in initiating the segregation of dLGN cells into layers.

\section{Potential mechanisms of laminar segregation}

In summary, our data indicate there are not significantly more growth cones or synapses in the interlaminar space than in the layers during the early period of laminar segregation. These results thus suggest that the segregation of dLGN cells into layers is not initiated by a selective development of neuropil elements at the laminar borders. This finding, however, does not by any means rule out a role of increasing neuropil in laminar segregation. Clearly, the increasing volume of neuropil as afferent pathways grow in, as dendrites and axon terminals develop, and as synaptic contacts are madc lcads to an incrcasc in interccllular space. The critical question is how an overall increase in neuropil (i.e., versus a directed increase at the laminar borders) could result in the segregation of dLGN cells into distinct layers.

One possible means of laminar segregation, although not directly related to an increase in neuropil, is that dLGN cells may actively migrate towards the center of individual layers. A second possibility is that cells destined to be in the same layer may have a greater affinity for, or adhesion to, each other than to cells in adjacent layers. Finally, cells destined to be in the same layer may be kept together by a outside force such as glial cell 
processes. In both of these latter possibilities, the overall increase in intercellular space due to expanding neuropil would lead to intercellular space formation along "faults" between layers where intercellular affinity is reduced or absent.

The possibility that cells migrate to the center of each layer is unlikely for 2 principal reasons. First; the cells do not appear migratory when examined ultrastructurally. Second, if migration were occurring, there should be an increased density of cells in each layer early in the period of laminar segregation. Although no quantitative data on this issue are presently available, light microscopic examination reveals that, while there may be an increase in density along the lateral border of layer 4 during laminar development, such an increase is not seen either along the medial border of layer 4 or in any of the other developing layers.

The other 2 possibilities, that the cells in a prospective layer have a higher affinity for each other than for cells in other layers or that the cells in a prospective layer are held together by outside constraints such as glia, remain plausible hypotheses. In either instance, the overall increase in intercellular space due to increasing volume of neuropil would lead directly to the formation of neuropil-filled spaces between individual cell layers. We are currently exploring both of these possibilities. One specific aspect of the interrelationship of adjacent neuronal somata is addressed in a companion paper (Brunso-Bechtold and Vinsant, 1988).

\section{References}

Boothe, R. G., W. T. Greenough, J. S. Lund, and K. Wrege (1979) A quantitative investigation of spine and dendrite development of neurons in visual cortex (area 17) of Macaca nemestrina monkeys. J. Comp. Neurol. 186: 473-490.

Brunso-Bechtold, J. K., and V. A. Casagrande (1981) Effect of bilateral enucleation on the development of layers in the dorsal lateral geniculate nucleus. Neuroscience 6:2579-2586.

Brunso-Bechtold, J. K., and V. A. Casagrande (1982) Early postnatal development of laminar characteristics in the dorsal lateral geniculate nucleus of the tree shrews. J. Neurosci. 2: 589-597.

Brunso-Bechtold, J. K., and V. A. Casagrande (1984) Development of layers in the dorsal lateral geniculate nucleus in tree shrew. In Contributions to Sensory Physiology, Vol. 8, W. D. Neff, ed., pp. 4177, Academic, New York.

Brunso-Bechtold, J. K., and S. L. Vinsant (1988) Cellular interrelationships during laminar segregation in the dorsal lateral geniculate nucleus. J. Neurosci. 8: 2693-2706.

Brunso-Bechtold, J. K., S. F. Florence, and V. A. Casagrande (1983) The role of retinogeniculate afferents in the development of connections between visual cortex and the dorsal lateral geniculate nucleus. Dev. Brain Res. 10: 33-39.

Bunge, M. B. (1973) Fine structure of nerve fibers and growth cones of isolated sympathetic neurons in cultures. J. Cell Biol. 56: 713-734.

Colonnier, M., and R. W. Guillery (1964) Synaptic organization in the lateral geniculate nucleus of the monkey. Z. Zellforsch. 62: 333355.

Cowan, W. M. (1970) Anterograde and retrograde transneuronal degeneration in the central and peripheral nervous system. Contemporary Research Methods in Neuroanatomy, W. J. M. Nauta and S. O. E. Ebbeson, eds., pp. 217-249, Springer-Verlag, New York.

Cusick, C. G., and J. H. Kaas (1982) Retinal projections in the adult and newborn grey squirrels. Dev. Brain Res. 4: 275-284.

Deitch, J. S., and E. W. Rubel (1984) Afferent influences on brain stem auditory nuclei of the chicken: Time course and specificity of dendritic atrophy following deafferentation. J. Comp. Neurol. 229: 66-79.

Del Cerro, M. P., and R. S. Snider (1968) Studies in the developing cerebellum. Ultrastructure of growth cones. J. Comp. Neurol. 133: 341-362.

Guillery, R. W. (1966) A study of Golgi preparations from the dorsal lateral geniculate nucleus of the cat. J. Comp. Neurol. 128: 21-50.
Guillery, R. W. (1969) The organization of synaptic interconnections in the laminae of the dorsal lateral geniculate nucleus of the cat. $Z$. Zellforsch. 96: 1-38.

Guillery, R. W., A.-S. LaMantia, J. A. Robson, and K. Huang (1985) The influence of retinal afferents upon the development of layers in the dorsal lateral geniculate nucleus of Mustelids. J. Neurosci. 5: 1370 1379.

Hajdu, F., R. Hassler, and G. Somogyi (1982) Neuronal and synaptic organization of the lateral geniculate nucleus of the tree shrew, Tupaia glis. Cell Tissue Res. 224: 207-223.

Hámori, J. (1973) The inductive role of presynaptic axons in the development of postsynaptic spines. Brain Res. 62: 337-344.

Hinds, J. A., and P. L. Hinds (1972) Reconstruction of dendritic growth cones in neonatal mouse olfactory bulb. J. Neurocytol. 1:169187.

Hopkins, W. G., and M. C. Brown (1984) Development of Nerve Cells and Their Connections, Cambridge U. P., New York.

Jones, E. G., and T. P. S. Powell (1969) Electron microscopy of synaptic glomeruli in the thalamic relay nuclei of the cat. Proc. R. Soc. London [Biol.] 172: 153-171.

Knyihár-Csillik, E., P. Rakic, and B. Csillik (1985) Fine structure of growth cones in the upper dorsal horn of the adult primate spinal cord in the course of reactive synaptoneogenesis. Cell Tissue Res. 239: 633-641.

LeVay, S. (1971) On the neurons and synapses of the lateral geniculate nucleus of the monkey, and the effects of eye enucleation. Z. Zellforsch. 113: 396-419.

Linden, D. C., R. W. Guillery, and J. Cucchiaro (1981) The dorsal lateral geniculate nucleus of the normal ferret and its postnatal development. J. Comp. Neurol. 203: 189-211.

Lund, R. D., and A. H. Bunt (1976) Prenatal development of central optic pathways in albino rats. J. Comp. Neurol. 165: 247-264.

Mason C. A. (1982a) Development of terminal arbors of retinogeniculate axons in the kitten. I. Light microscopical observations. Neuroscience $7: 541-559$.

Mason, C. A. (1982b) Development of terminal arbors of retinogeniculate axons in the kitten. II. Electron microscopial observations. Neuroscience 7: 561-582.

Mason, C. A. (1985a) Growing tips of embryonic cerebellar axons in vivo. J. Neurosci. Res. 13: 55-73.

Mason, C. A. (1985b) How do growth concs grow? Trends Neurosci. 8: $304-308$

Mason, C. A., and J. A. Robson (1979) Morphology of retinogeniculate axons in the cat. Neuroscience 4: 79-98.

Michel, A. E., and L. J. Garey (1984) The development of dendritic spines in the human visual cortex. Human Embryol. 3: 223-227.

Morest, D. K. (1969) The growth of dendrites in the mammalian brain. Z. Anat. Entwickl.-Gesch. 128: 290-317.

Norlander, R. H., and M. Singer (1982) Morphology and position of growth cones in the developing Xenopus spinal cord. Dev. Brain Res. 4: $181-193$.

Peusner, K. D., and D. K. Morest (1977) Neurogenesis in the nucleus vestibularis tangentialis of the chick embryo in the absence of the primary afferent fibers. Neuroscience 2: 253-270.

Pfenninger, K. II., and R. P. Bunge (1974) Freeze fracturing of nerve growth cones and young fibers. A study of the developing plasma membrane. J. Cell Biol. 63: 180-196.

Kakic, P. (1977) Genesis of the dorsal lateral geniculate nucleus in the rhesus monkey: Site and time of origin, kinetics of proliferation, routes of migration and pattern of distribution of neurons. J. Comp. Neurol. 176: 23-52.

Reh, T. A., and M. Constantine-Paton (1985) Growth cone-target interactions in the frog retinotictal pathway. J. Neurosci. Res. 13:89100.

Robson, J. A., and C. A. Mason (1979) The synaptic organization of terminals traced from individual labeled retinogeniculate axons in the cat. Neuroscience 4: 99-112.

Shatz, C. J. (1983) The prenatal development of the cat's retinogeniculate pathway. J. Neurosci. 3: 482-499.

Shatz, C. J., and P. A. Kirkwood (1984) Prenatal development of functional connections in the cat's retinogeniculate pathway. J. Neurosci. 4: 1378-1397.

Skoff, R. P., and V. Hamburger (1974) Fine structure of dendritic and axonal growth cones in embryonic chick spinal cord. J. Comp. Neurol. 153: $107-148$ 
Smith, D. J. Z. (1981) Organization and development of brain stem auditory nuclei of the chicken: Dendritic development in $N$. laminaris. J. Comp. Neurol. 203: 309-333.

So, K. F., G. E. Schneider, and P. D. Frost (1978) Postnatal development of retinal projections to the lateral geniculate body in Syrian hamsters. Brain Res. 142: 343-352.

Sretavan, D. W., and C. J. Shatz (1984) Prenatal development of individual retinogeniculate axons during the period of segregation. Nature 308: 845-848.

Sretavan, D. W., and C. J. Shatz (1986) Prenatal development of retinal ganglion cell axons: Segregation into eye-specific layers within the cat's lateral geniculate nucleus. J. Neurosci. 6: 234-251.

Steward, O., and P. M. Falk (1986) Protein-synthetic machinery at postsynaptic sites during synaptogenesis: A quantitative study of the association between polyribosomes and developing synapses. J. Neurosci. 6: $412-423$.

Sutton, J. K., and J. K. Brunso-Bechtold (1988) The roles of specificity and competition in the formation of a laminated colliculogeniculate projection. J. Neurosci. 8: 435-444.

Szentágothai, J. (1963) The structure of the synapse in the lateral geniculate body. Acta Anat. (Basel) 55: 166-185.

Szentágothai, J. (1970) Glomerular synapses, complex synaptic arrangements and their operational significance. In The Neurosciences, 2nd Study Program, F. O. Schmitt, ed., pp. 427-443, Rockefeller U. P., New York.
Szentágothai, J., J. Hámori, and T. Tömböl (1966) Degeneration and electron microscope analysis of the synaptic glomeruli in the lateral geniculate body. Exp. Brain Res. 2: 283-301.

Tennyson, V. M. (1970) The fine structure of the axon and growth cone of the dorsal root neuroblast of the rabbit embryo. J. Cell Biol. 44: 62-79.

Vaughn, J. E., C. K. Hendrickson, and J. A. Grieshaber (1974) A quantitative study of synapses on motor neuron dendritic growth cones in developing mouse spinal cord. J. Cell Biol. 60: 664-672.

Vinsant, S. L., and J. K. Brunso-Bechtold (1986) Size and distribution of growth cones in the developing lateral geniculate nucleus. Soc. Neurosci. Abstr. 12: 590.

Williams, R. W., M. J. Bastiani, B. Lia, and L. M. Chalupa (1986) Growth cones, dying axons, and developmental fluctuations in the fiber population of the cat's optic nerve. J. Comp. Neurol. 246: 3269.

Wong-Riley, M. T. T. (1972a) Neuronal and synaptic organization of the normal dorsal lateral geniculate nucleus of the squirrel monkey, Saimiri sciureus. J. Comp. Neurol. 144: 25-60.

Wong-Riley, M. T. T. (1972b) Terminal degeneration and glial reactions in the lateral geniculate nucleus of the squirrel monkey after eye removal. J. Comp. Neurol. 144: 61-92.

Yamada, K. M., B. S. Spooner, and N. K. Wessells (1974) Ultrastructure and function of growth cones and axons in cultured nerve cells. J. Cell Biol. 49: 614-635. 